\title{
Stochastic sawtooth reconnection in ASDEX Upgrade
}

\author{
V. Igochine ${ }^{1}$, O. Dumbrajs ${ }^{2,3}$, H. Zohm ${ }^{1}$, A. Flaws ${ }^{1}$ and ASDEX Upgrade Team ${ }^{1}$ \\ ${ }^{1}$ MPI für Plasmaphysik, Euratom-Association, D-85748 Garching, Germany \\ ${ }^{2}$ Helsinki University of Technology, Association Euratom-Tekes, P.O.Box 2200, FIN-02015 HUT, Finland \\ ${ }^{3}$ Institute of Solid State Physics, Association Euratom-University of Latvia, Kengaraga Street 8, LV-1063, Riga, Latvia
}

\begin{abstract}
In this paper we investigate non-complete Sawtooth reconnection in ASDEX Upgrade tokamak. Such reconnection phenomena are associated with internal $\mathrm{m} / \mathrm{n}=1 / 1$ kink mode which does not vanish after the crash phase (as would be the case for complete reconnection). It is shown that this sawtooth can not be fully described by pure $\mathrm{m} / \mathrm{n}=1 / 1$ mode and that higher harmonics play an important role during the Sawtooth crash phase. We employ the Hamiltonian formalism and reconstructed perturbations to model incomplete Sawtooth reconnection. It is demonstrated that stochastization appears due to excitation of low- order resonances which are present in the corresponding q-profiles inside the $q=1$ surface which reflects the key role of the $q_{0}$ value. Depending on this value two completely different situations are possible for one and the same mode perturbations: (i) the resonant surfaces are present in q-profile leading to stochasticity and sawtooth crash $\left(q_{0} \approx 0.7 \pm 0.1\right)$; (ii) the resonant surfaces are not present which means no stochasticity in the system and no crash event $\left(q_{0} \approx 0.9 \pm 0.05\right)$. Accordingly central safety factor value is always less than unity in case of non-complete sawtooth reconnection. Our investigations show that stochastic model agrees well with experimental observations and can be proposed as a promising candidate for explanation of the sawtooth reconnection.
\end{abstract}

\section{Introduction}

Sawtooth oscillations are a periodic relaxation process of the plasma temperature, density and other plasma parameters in the central region of a tokamak [1]. This relaxation process is associated with internal $(m, n)=(1,1)$ kink mode. Investigation of sawtooth crashes in ASDEX Upgrade shows that in many cases the magnetic reconnection is not complete [2]. This means that the $(1,1)$ mode survives the crash and slowly decays after it. Such decay includes few tens of rotation periods of the mode what is much longer than the crash time itself (hundreds of microseconds). This non-complete reconnection is not a 
unique feature of ASDEX Upgrade tokamak and was observed also on other tokamaks (TEXTOR, Tore Supra, etc.) [3]. There exist several theories which address the sawtooth phenomenon. Some of them require flattening of the safety factor profile and $q_{0} \geq 1$ after the crash (full reconnection model [6], quasi-interchange model [7]). These models are in contradiction with experimental measurements after the sawtooth crash, because in case of non-complete reconnection one observes instabilities located at $q=1$ resonant surfaces after the crash phase. Thus, the main condition is not fulfilled and $q_{0}$ always less then unity. Other models [8] assume that the sawtooth is not triggered by the $m=1$ helical mode and allow that in the central region the safety factor $q$ is lower than unity. At the same time, experimental measurements show that the crash events always correspond to the maximal amplitude of the internal kink instability. Thus, such theories cannot describe sawtooth events observed in ASDEX Upgrade. In this paper, we employ the stochasticity hypothesis which was proposed to explain the sawtooth phenomenon without a full reconnection $[9,10]$ assuming the interaction of the $(1,1)$ mode with other periodicities and utilizing on the Hamiltonian formalism. The work consists of five sections. In section 2, we analyse experimental data and reconstruct the structure of the mode. The basic information about the Hamiltonian formalism and its use in the present study are described in section 3. In section 4 we present the results of calculations and demonstrate the key role played by the central q-value in the partial reconnection. Finally, in section 5 we summarize the main results and outline guidelines for future work.

\section{Reconstruction of the MHD modes}

As was mentioned in the previous section, reconnection during sawtooth crashes in ASDEX Upgrade in many cases is not complete as seen in Fig.1. The spectral analysis of the mode before and after the crash is also shown in Fig.1. 

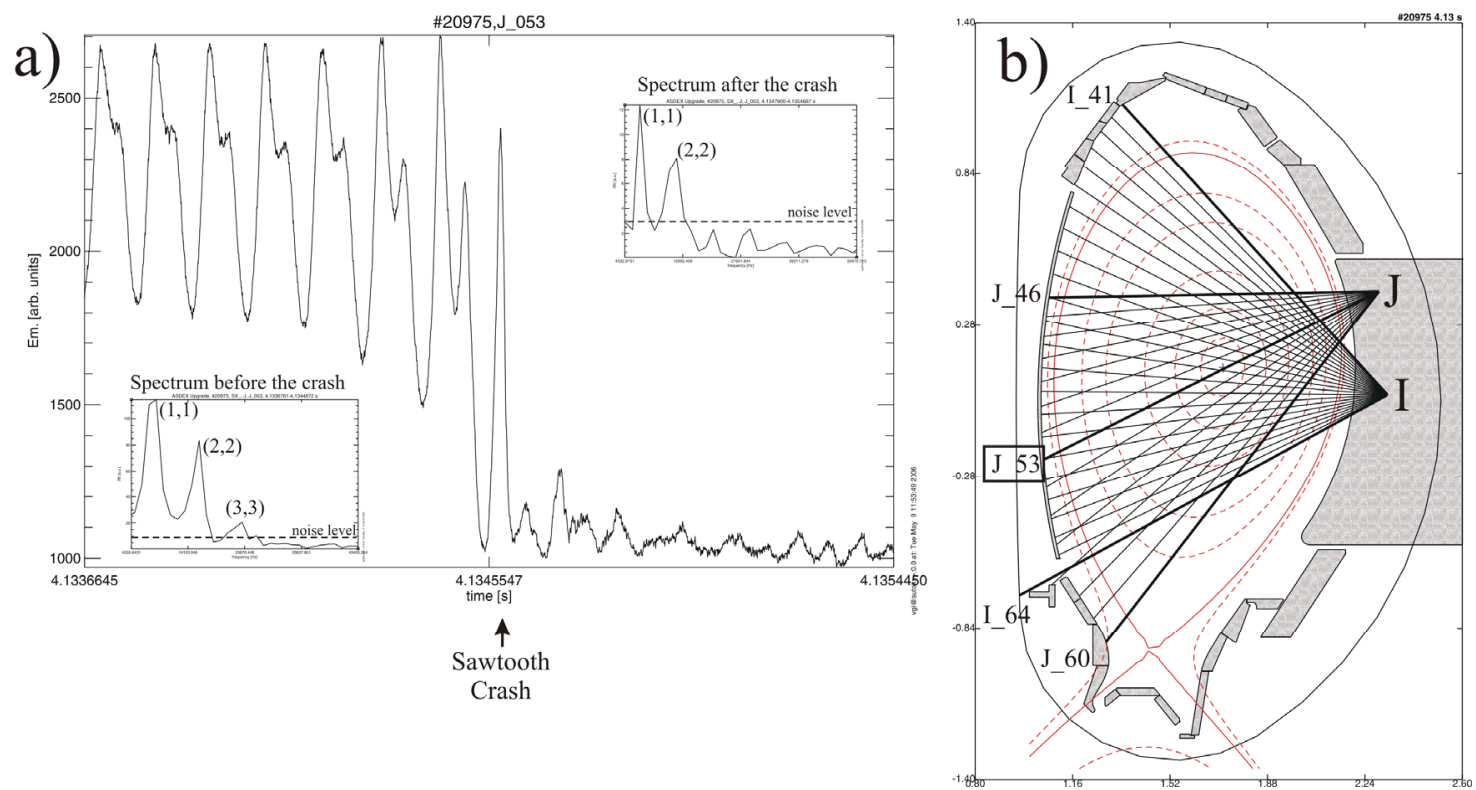

Figure 1. a) Central SXR signal during sawtooth crash and its spectra before (t=4.13367614.1344872s) and after $(t=4.13479-4.1354667 s)$ crash for central SXR line J_53. b) Some SXR lines of sight with central line $\mathbf{J}$ 53. (Total number of $S X R$ lines is 128.)

The analysis of this signal leads to two important conclusions. First, the mode survives the crash and subsequently slowly decays, which means that the reconnection is not complete during the crash. Second, the sawtooth oscillations cannot be fully described by a single $(1,1)$ helicity. One has to include at least the second $(2,2)$ component before and after the crash. The small third component $(3,3)$ is also seen before the crash. In other words, instead of representing a displacement in the standard form

$$
\xi_{\text {Sawtooth }}=\xi_{(1,1)}(\rho) \cdot \cos (\theta+\varphi)
$$

where $\theta$ is the poloidal angle, $\varphi$ is the toroidal angle and $\xi(\rho)$ is radial structure of the displacement, we use the following expression to describe the sawtooth crash with maximal accuracy:

$$
\xi_{\text {Sawtooth }}=\xi_{(1,1)}(\rho) \cdot \cos (\theta+\varphi)+\xi_{(2,2)}(\rho) \cdot \cos (2 \theta+2 \varphi)+\xi_{(3,3)}(\rho) \cdot \cos (3 \theta+3 \varphi)
$$

All these harmonics are phase locked (i.e. $f_{(1,1)}=f_{(2,2)} / 2=f_{(3,3)} / 3$ ) and the temporal behaviour due to rotation is trivial. It can be clearly seen in figure 2 that these components appear just before the sawtooth crash. 


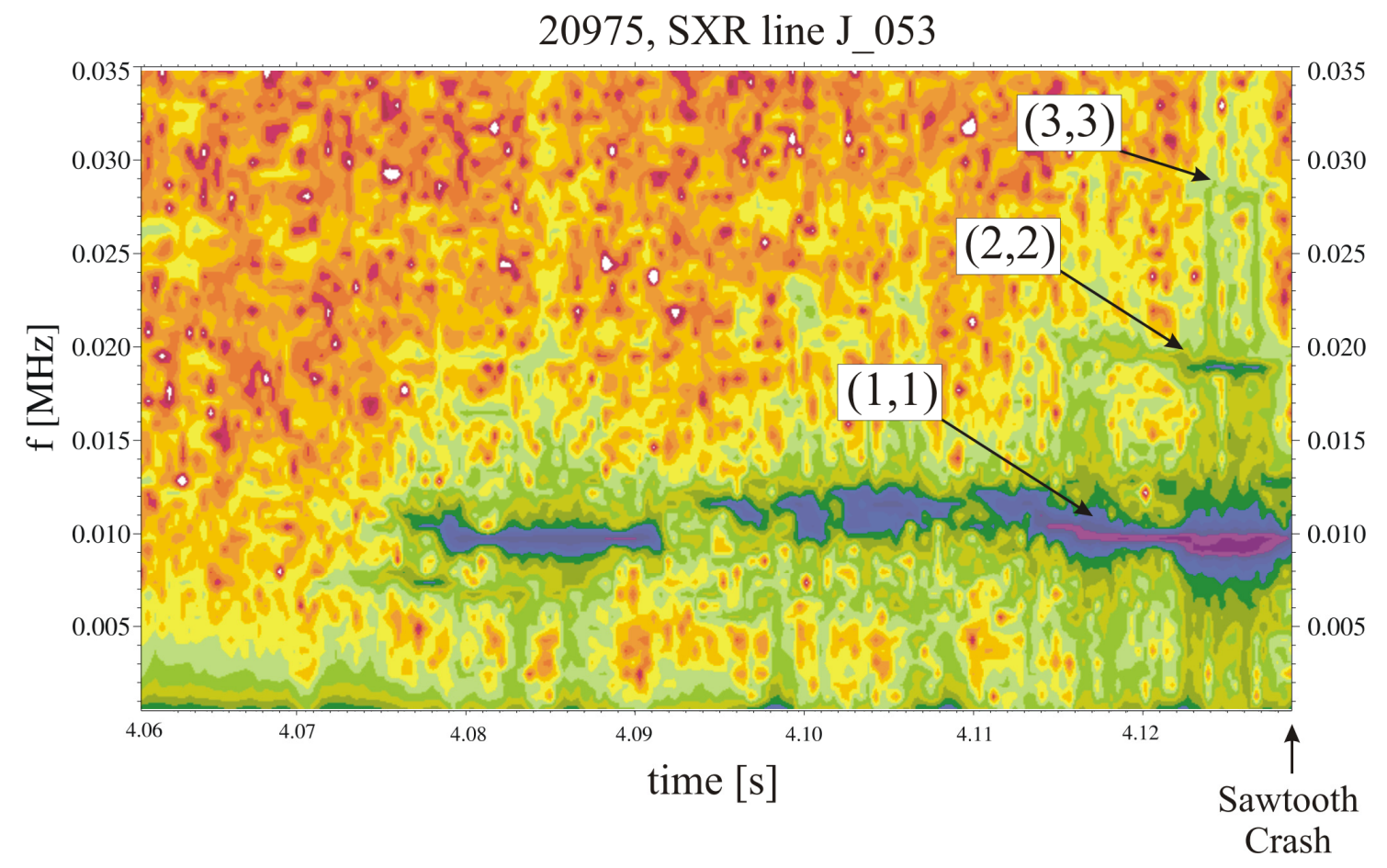

Figure 2. Spectrogram of the SXR signal before the sawtooth crash. The primary $(1,1)$ mode exists in the spectrogram well before the crash. The second and third components appear just before the crash.

The second and third components indicate an importance of mode coupling in the sawtooth evolution before the crash. Between the crashes only the dominant $(1,1)$ component is present. Simple modelling of the experimental signal from the central SXR line (J_053, figure 1) helps us to understand the influence of the second harmonic on the primary $(1,1)$ mode. This model assumes the existence of two dominant modes, with double frequency for the second harmonic (figure 3). We can see that the main features of the signal can be reproduced by this simple model. Before the crash, the relative amplitude of the second harmonic is only by factor two or three smaller than the first one. This model reproduces the main features of the signal (higher harmonics and more precise fitting of the growth rates are necessary for complete description of the signal). It is interesting to note that higher m-components during Sawteeth were also observed in other tokamaks $[3,4]$. 

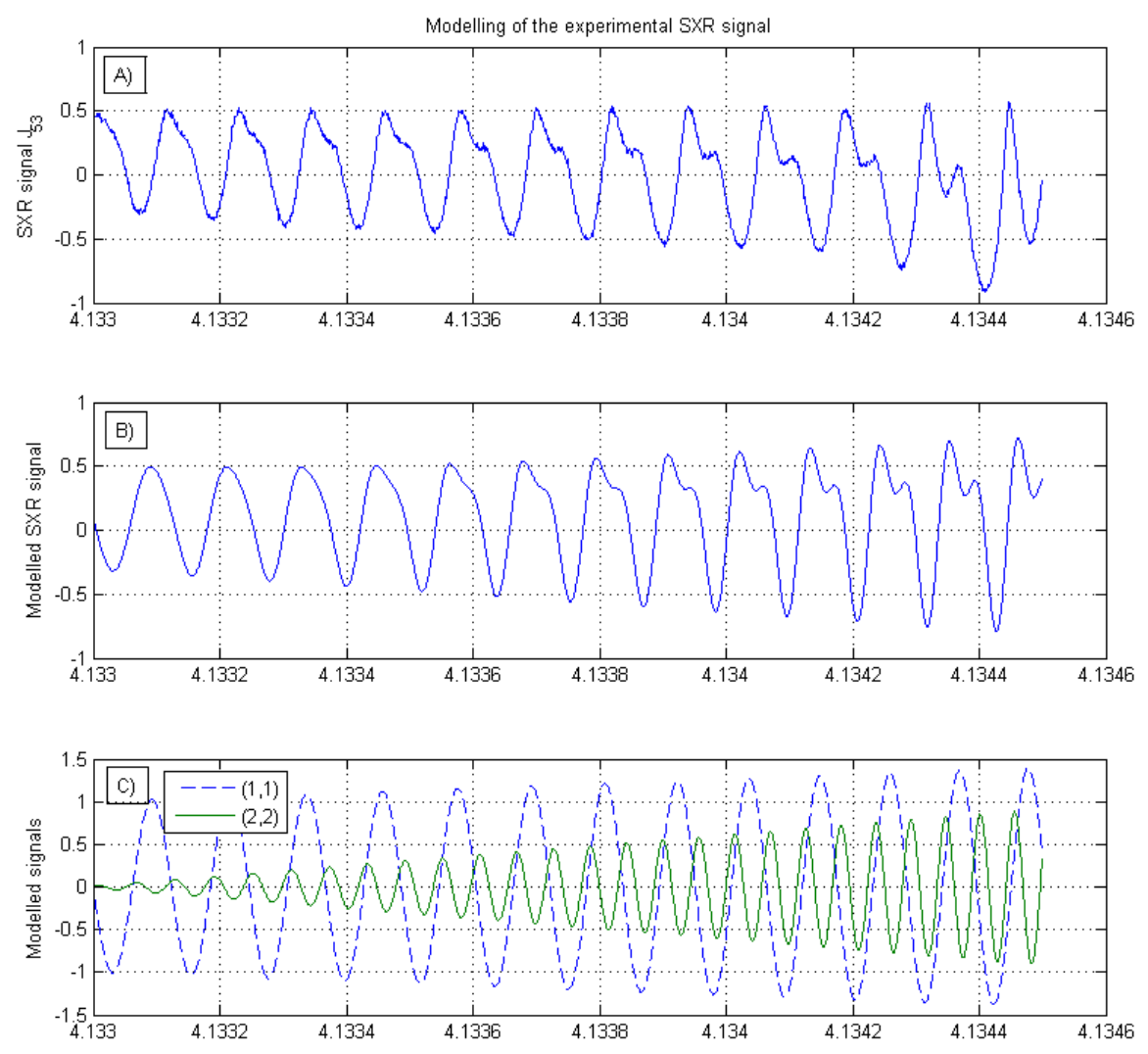

Figure 3. Modelling of the experimental signal are shown. A) Experimental signal for SXR line J_53 (the same as in figures 1 and 2 ) is shown. B) Modelled signal is a superposition of two sinusoidal signals from the next figure. C) The signals which is used for superposition in figure (B).

As a next step we reconstruct the radial parts of the displacement eigenfunctions (Eq. 2) just before the crash. Here we apply the MHD-Interpretation code (MHD-IC) to reconstruct the shapes and relative amplitudes of the displacements [5]. The code simulates experimental observations related to a given plasma perturbation for several diagnostics (magnetic measurements, ECE and Soft X-ray cameras), accounting for real plasma geometry and for measured plasma parameters.

Sawtooth oscillations are strongly core localized phenomena. In such a situation, magnetic measurements are affected by the $(2,1)$ harmonic which is weak but is positioned much closer to the plasma boundary. This $(2,1)$ mode is coupled to $(1,1)$ mode at the low field side which gives several problems for interpretation of the magnetic 
signals (for example phase folding problem which is discussed in Ref.[11]). ECE measurements are limited by the density cut-off. Thus, SXR cameras are our main source of information for sawtooth events. Additional difficulties are related to the fact that only a few SXR lines from each camera come through the $\mathrm{q}=1$ resonant surface.

MHD-IC code models experimental signal for given plasma perturbations. Detailed information about the mode structure can be gained if the perturbed part of the SXR signal is separated from the background. The main tools for our analysis are the fast Fourier transformation (FFT) and the singular value decomposition (SVD). We use these algorithms to separate mode perturbations from the background and fit to the experimentally measured perturbations [5]. The best fit was obtained for the displacement eigenfunctions shown in figure 4.

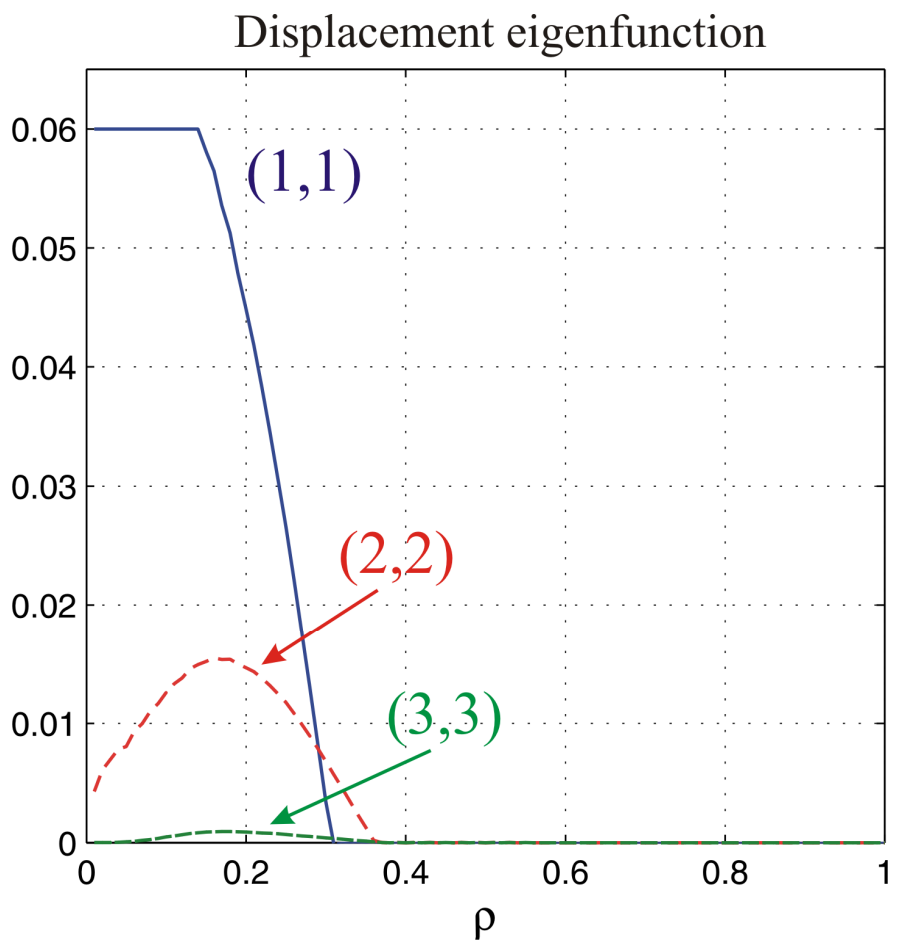

Figure 4. Displacement eigenfunctions for first three components before the sawtooth crash. Amplitudes of the displacements are in meters.

In this case a good agreement between predictions from MHD-IC code and experimental results were obtained. Here we show comparison for FFT amplitudes and SVD eigenvectors (figures 5 and 6). Such a comparison allows one to find shapes and relative amplitudes of the $(1,1)$ and $(2,2)$ modes. Identification of the $(3,3)$ radial structure is not 
possible, because the signal for this mode is very close to the noise level (figure 1). We use for this mode a shape similar to the $(2,2)$ mode and estimate the relative size of the FFT amplitudes to the experimental values. The best fit was obtained by manual variation of shape and amplitudes of the displacements.
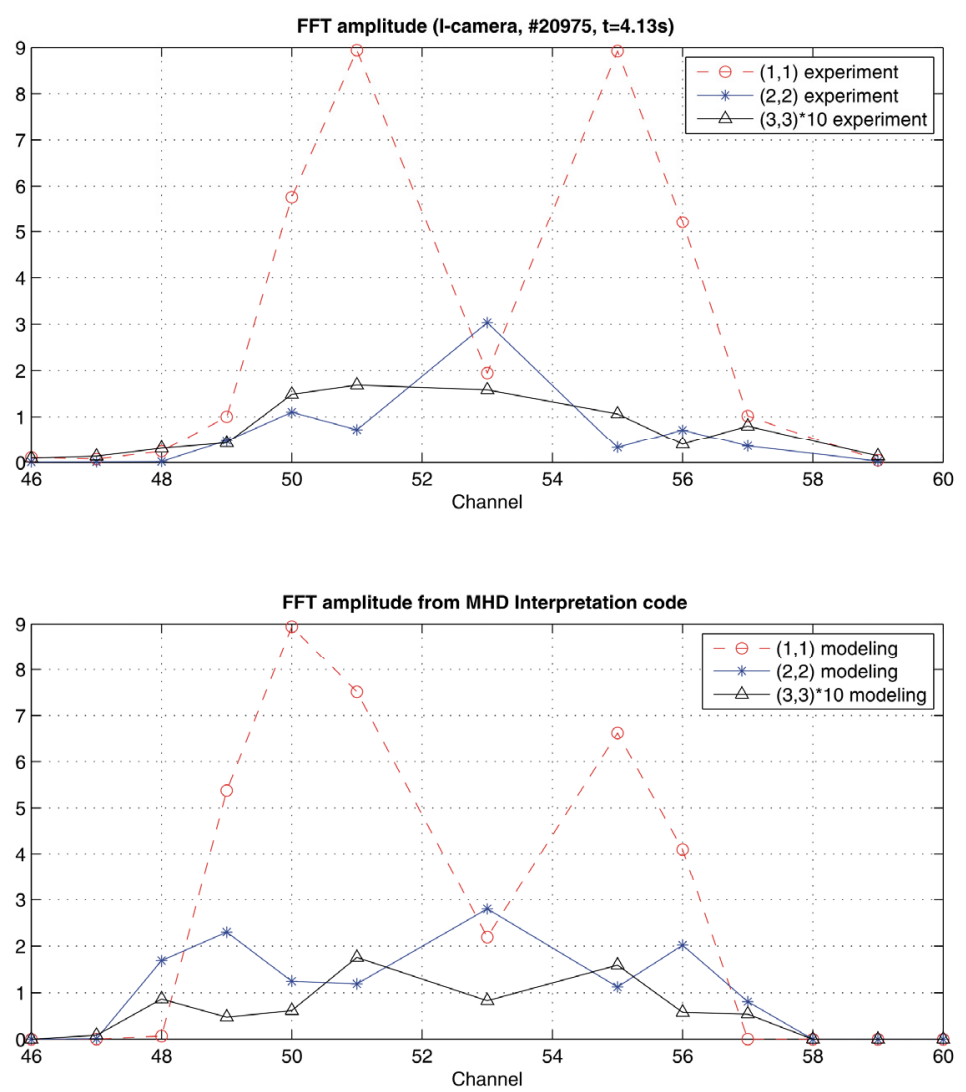

Figure 5. Comparison of the experimental FFT amplitude with calculated FFT amplitude from MHD-IC code. (Positions of the SXR lines for I-camera are shown in figure 1.) 

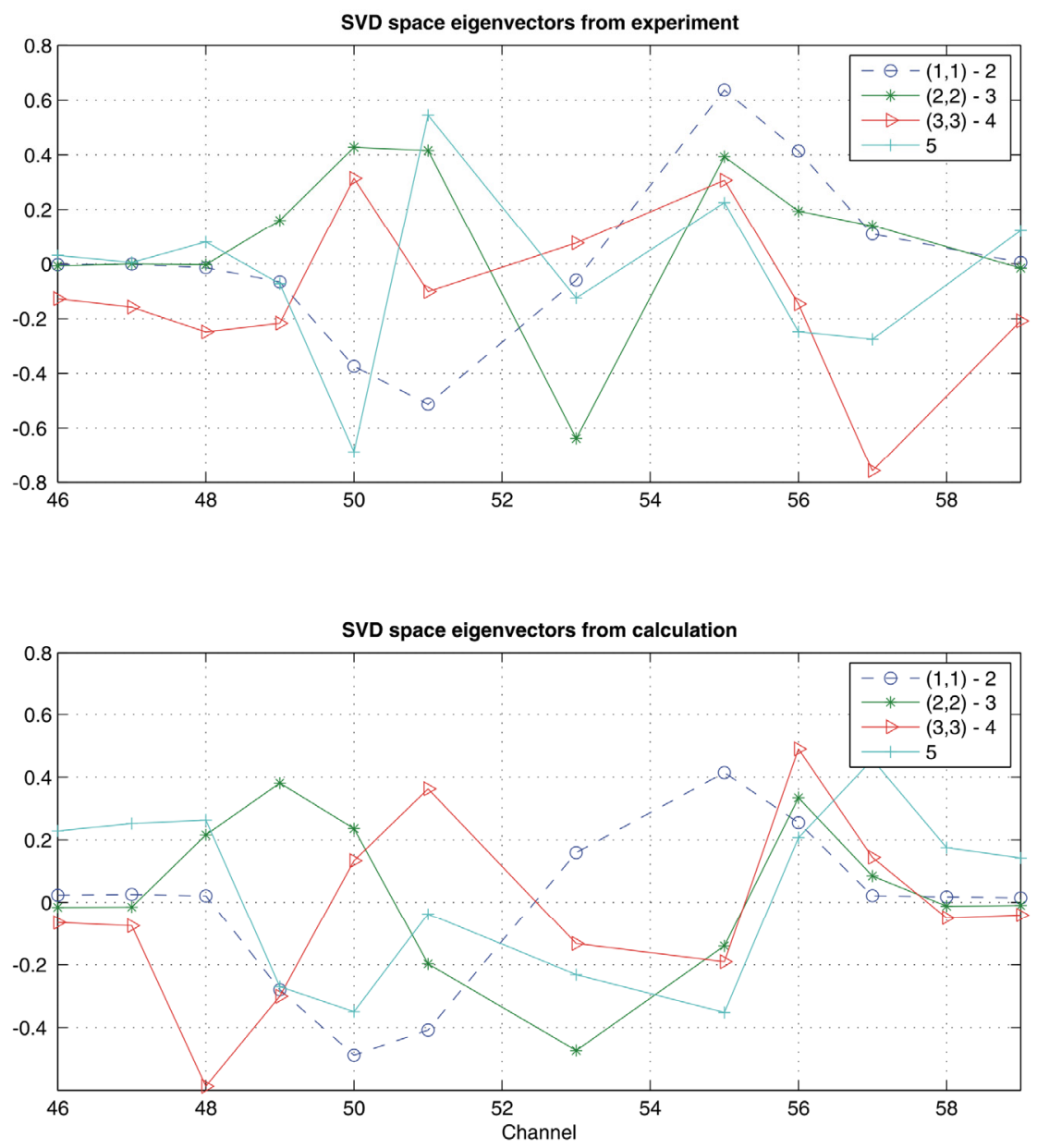

Figure 6. SVD eigenvectors for the same sawtooth as in figures 4 and 5 . The first eigenvector is not shown because it contains equilibrium part of the signal. Second eigenvector corresponds to $(1,1)$ component. Third and fourth eigenvectors represent $(2,2)$ and $(3,3)$ modes respectively. (Positions of the SXR lines for I-camera are shown in figure 1.)

The absolute value of the $(1,1)$ displacement was estimated from few points of ECE temperature measurements inside Sawtooth inversion radius to be about $6 \mathrm{~cm}$. Relative amplitude of the displacements can be estimated from figure 4:

$$
\frac{\max \xi_{(1,1)}}{\max \xi_{(2,2)}}=4-5
$$

The $q=1$ resonant surface is at about $\rho \approx 0.25-0.3$ (see figure 4, approximate boundary for $(1,1)$ displacement). These results can be compared with the tomography reconstruction which employs the maximum entropy method [12] and the rotational symmetry [13] of the MHD modes. Both options strongly improve the quality of 
reconstruction and make it possible to reconstruct the two $(1,1)$ and $(2,2)$ modes separately (figure 7). The positions of the modes from differential rotation tomography are the same as those obtained by means of the MHD-IC code $(\rho \approx 0.25-0.3)$. Determination of the eigenfunctions shapes and absolute values requires derivative of the emissivity profile which results in large error bars for these quantities.
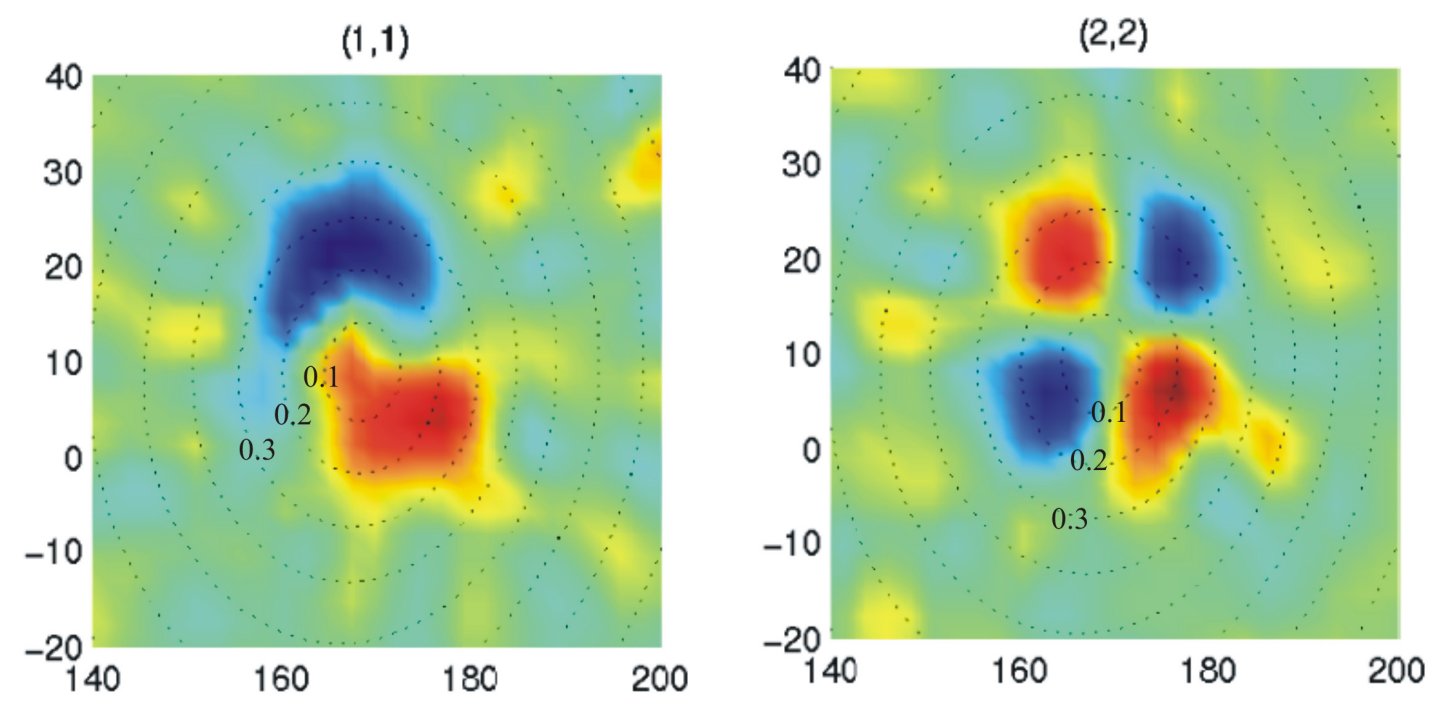

Figure 7. Tomography reconstruction of the $(1,1)$ and $(2,2)$ modes. These modes have different frequencies and can be reconstructed separately. Tomography reconstruction employs maximum entropy method [12] and rotational symmetry of the mode [13].

\section{Hamiltonian formalism and stochasticity}

The mapping technique for Hamiltonian problems has become popular during the last years due to higher performance compared to the direct integration methods and due to conservation of the flux property which is not the case for integration [14]. The sawtooth phenomenon was analysed by means of this technique for the first time in [15]. However in that work a single perturbation parameter was used which does not take into account the experimental information about the structure of perturbations. In this work we apply a more advanced method which allows us to specify experimental perturbations for Hamiltonian formalism. In the formalism the equations for magnetic field lines take the Hamiltonian form

$\frac{d \psi}{d \varphi}=-\frac{\partial H}{\partial \vartheta}, \quad \frac{d \vartheta}{d \varphi}=\frac{\partial H}{\partial \psi}$, 
where $\psi=\frac{r^{2}}{2 a^{2}}$ is a toroidal magnetic flux canonically conjugated to the poloidal angle $\vartheta$, $\varphi$ is a toroidal angle, and $a$ is a minor radius of the plasma (50 $\mathrm{cm}$ at ASDEX Upgrade).

The Hamiltonian $H$

$H=H_{0}(\psi)+H_{1}(\psi, \vartheta, \varphi)$

can be represented as a sum of the unperturbed flux

$H_{0}(\psi)=\int \frac{d \psi}{q(\psi)}$

and the perturbed part of the flux

$H_{1}(\psi, \vartheta, \varphi)=\sum_{m, n} H_{m n}(\psi) \cos \left(m \vartheta-n \varphi+\chi_{m n}\right)$

Here $q(\psi)$ is the safety factor characterizing the winding of the magnetic field lines, $H_{m n}(\psi)$ is the perturbation Hamiltonian which corresponds to the perturbations of the modes $(\mathrm{m}, \mathrm{n})$ with the phases $\chi_{m n}$. For our purposes we have chosen the symmetric symplectic mapping derived on the basis of the Hamilton-Jacobi method [16]. It is obvious that practical implementation of the mapping method requires knowledge of the safety factor and of the perturbation Hamiltonian. The relation between the displacement and the perturbed flux follows from the Ohm's law:

$$
\Psi_{\text {pert }}(r)=\xi(r) \frac{r B_{z}}{R_{0}}\left(\frac{1}{q(r)}-\frac{n}{m}\right)
$$

where, $B_{z}$ is the primary toroidal magnetic field and $R_{0}$ is the major plasma radius. Such flux is exactly the perturbed part of the Hamiltonian which has the following form in the Hamiltonian coordinates:

$$
H_{m, n}(\psi)=\xi_{m, n}(\psi) \frac{a \sqrt{2 \psi} B_{z}}{R_{0}}\left(\frac{1}{q(\psi)}-\frac{n}{m}\right)
$$

Accurate determination of the central q-profile is not possible in ASDEX Upgrade to the degree needed here and we will investigate its influence in the next section. 


\section{Influence of different parameters on stochastization}

In this section we apply the Hamiltonian mapping technique and address the following questions: (i) role of the higher harmonics during partial sawtooth crash, (ii) role of the qprofile, (iii) influence of the perturbation amplitudes. For our first calculations we consider a smooth q-profile with central value 0.7 . Position of the $\mathrm{q}=1$ resonant surface is about $\rho=0.3$ which corresponds to $\psi=0.045$ in the Hamiltonian coordinates. We show the influence of the second and third harmonics in figure 8.
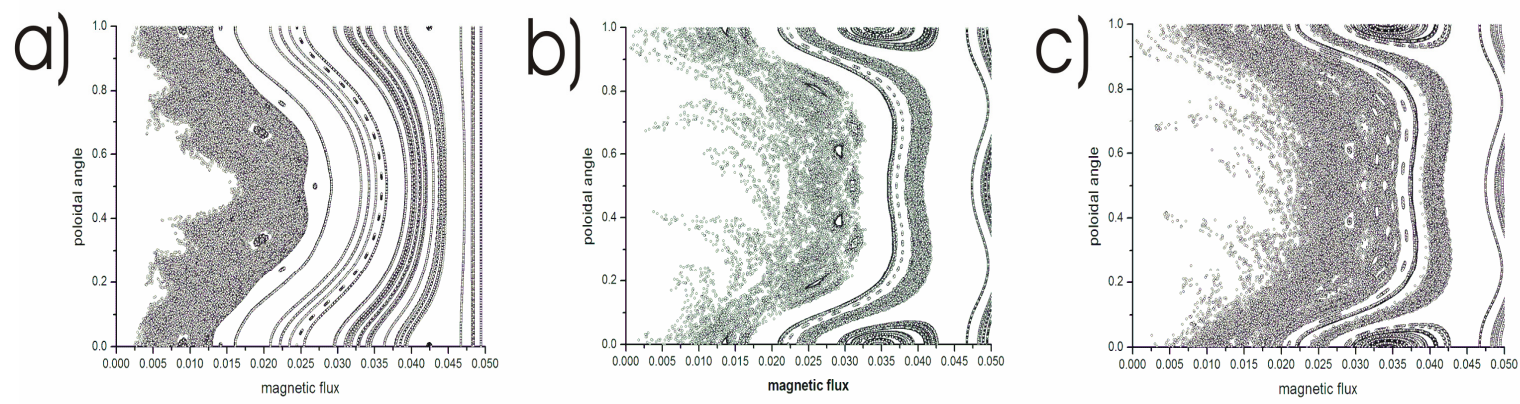

Figure 8. Poincare plots for displacements from figure 4: a) only $(1,1)$, b) $(1,1)$ and $(2,2)$, c) $(1,1),(2,2)$ and $(3,3)$. The $(3,3)$ component only slightly increases the stochasticity compare to the case with $(1,1)+(2,2)$. 
One can clearly see that even the relatively small amplitudes of $(2,2)$ and $(3,3)$ modes considerably increase the stochastic region. In the presence of all three modes, a large stochastic region develops inside the $\mathrm{q}=1$ resonance surface, with exception of the islands themselves. One would expect that such a stochastization destroys the confinement inside the $\mathrm{q}=1$ resonance surface and leads to sawtooth crash. At the same time, the island structure is not altered by the stochastization and the mode survives the crash. It should be remembered that the small $(2,2)$ and $(3,3)$ perturbations are located in the same spatial region as the main $(1,1)$ perturbation (figure 4$)$. This leads to a much more effective destabilisation of all low order resonances inside the $\mathrm{q}=1$ surface compared to the case of two resonances located at the neighbouring resonant surfaces.

As a next step we investigate influence of the safety factor profile (figure 9).
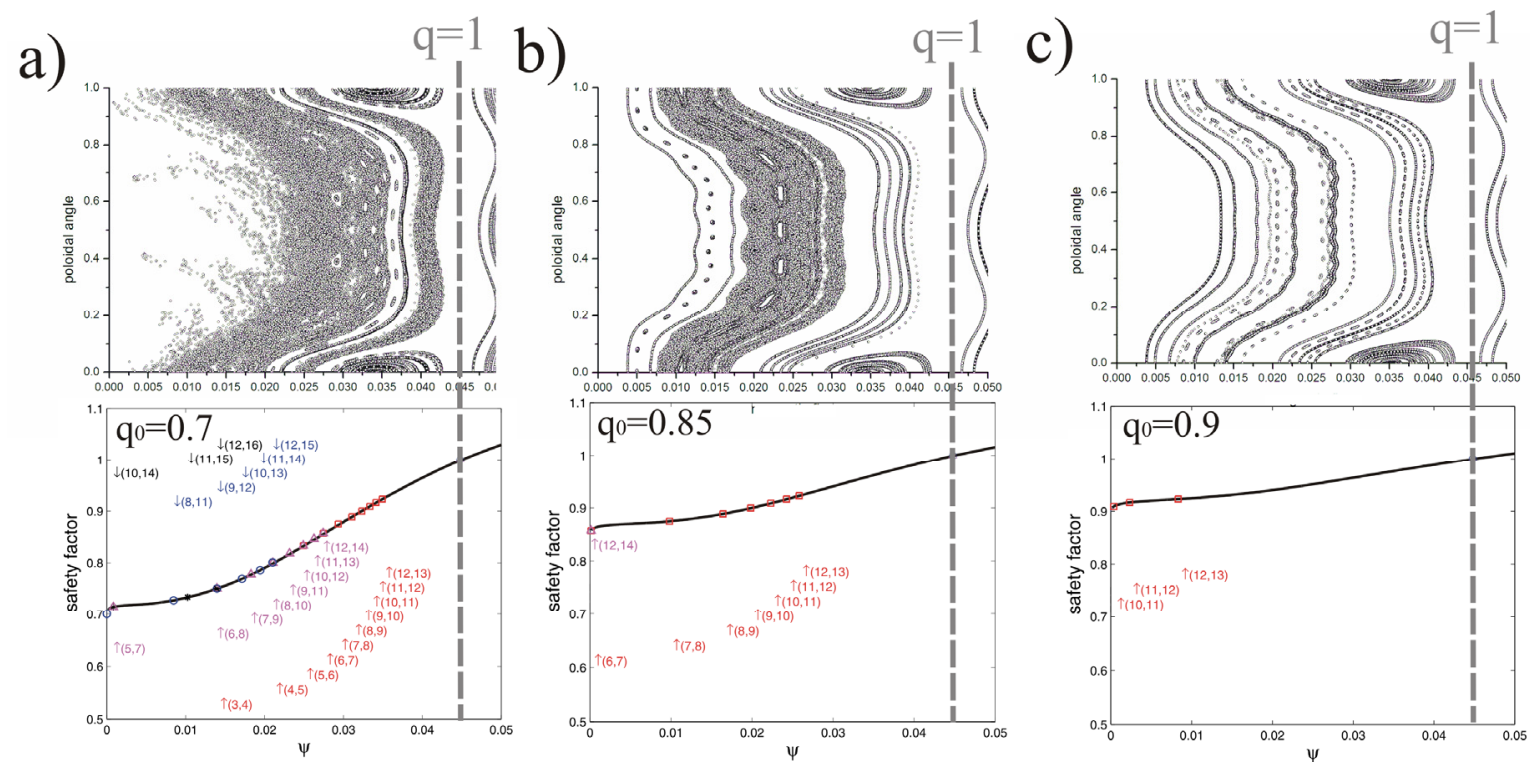

Figure 9. Poincare plots for the same perturbations $(1,1)+(2,2)+(3,3)$ as in figure 4 but different safety factor profiles. Note that stochastization strongly depends on the existence of the low-order rational surfaces which are marked on safety factor curves. a) central q-value is 0.7 ; b) central q-value is 0.85 ; c) central $q$-value is 0.9

Here Poincare plots are shown together with the corresponding safety factor profiles. The low-order rational surfaces are marked on safety factor curves. It can be clearly seen that with increasing $q_{0}$ stochastic region is reduced and vanishes completely for $q_{0}=0.9$. At the same time, a strong reduction of the existing low-order rational surfaces in the qprofile can be observed. These two observations provide a key for understanding the 
partial sawtooth reconnection. For conventional tokamak scenarios a monotonically increasing q-profile is characteristic. Thus, $q_{0}$ determines the number of the low-order rational surfaces for a particular q-profile. Stochastization requires the existence of several low-order resonant surfaces which can be excited by the overlapping $(1,1),(2,2)$ and $(3,3)$ resonances. Without these resonant surfaces, the $(2,2)$ and $(3,3)$ perturbations only slightly modify the shape of the $(1,1)$ mode and the system is not stochastic at all (figure 9c)! It is interesting to note that for the considered experimental plasma perturbations the critical value for avoiding stochastization is $q_{0}=0.90 \pm 0.05$ (smaller than unity!). The critical $q_{0}$ value to create a stochastic zone is about $0.7 \pm 0.1$. In order to estimate the error bars for $q_{0}$, a set of calculations with different safety factor profiles and fixed perturbations amplitudes (see figure 4) were done. Threshold of the stochasticity is nicely seen from the result Poincare plots. Thus, we conjecture that during the sawtooth cycle $q_{0}$ changes between 0.7 and 0.9 , which is in a very good agreement with the qprofile measurements during the Sawtooth crashes in other tokamaks $\left(0.75<q_{0}<0.95\right.$, Levinton on TFTR [17]; $0.7<q_{0}<0.85$, Yamada on TFTR [18]; $q_{0} \approx 0.77 \pm 8 \%, \mathrm{H}$. Soltwisch on TEXTOR [19]). Exact values of $q_{0}^{\max }$ (no stochastization inside $\mathrm{q}=1$ ) and $q_{0}^{\min }$ (complete stochastization inside $\mathrm{q}=1$ ) depend also on absolute values of the perturbation as shown in Fig.10. One can see that small amplitudes (less than $3 \mathrm{~cm}$ for $(1,1)$ mode) are not sufficient to create 
stochasticity.
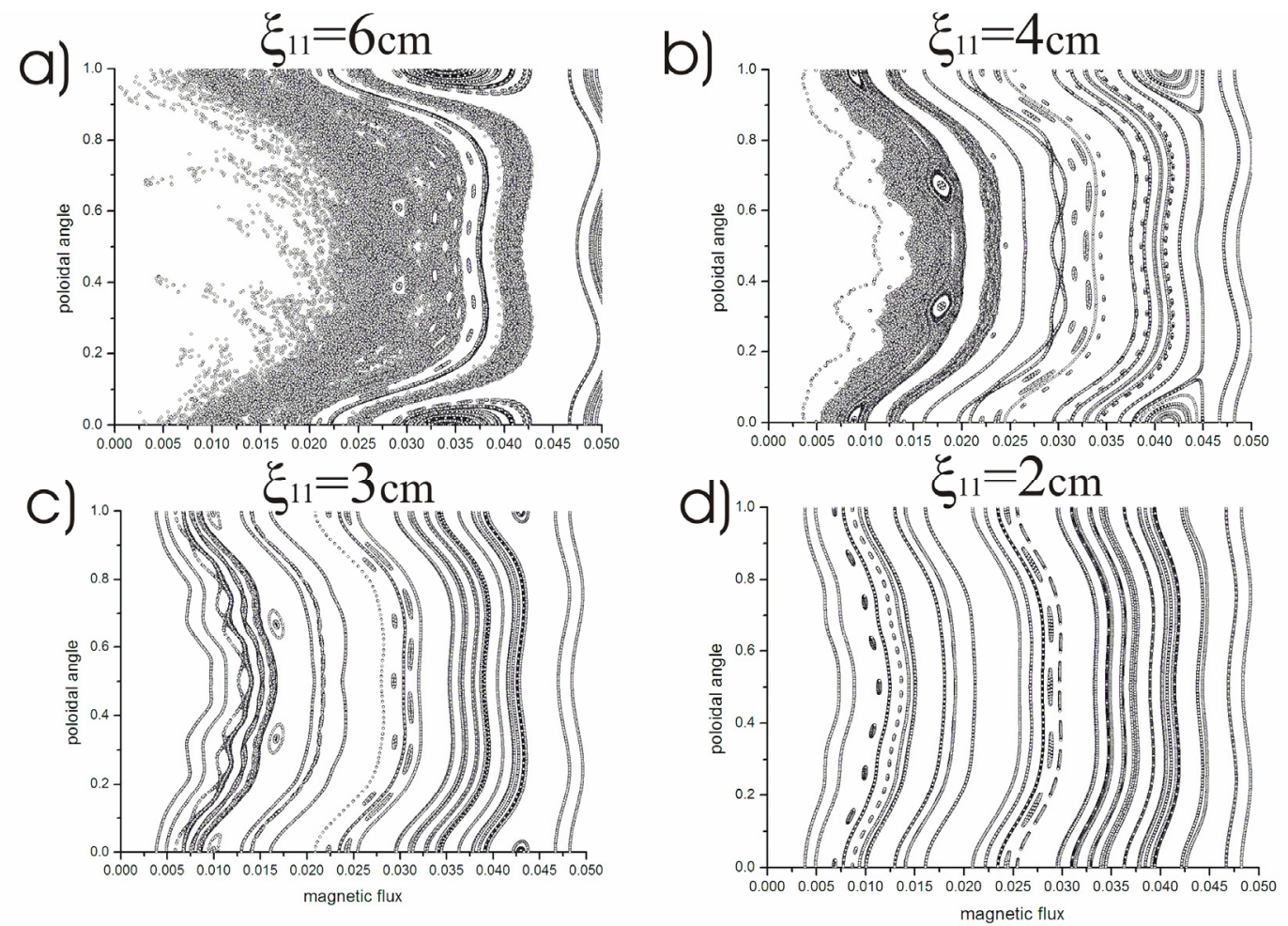

Figure 10. Influence of the absolute amplitude of the perturbations on stochasticity. Safety factor

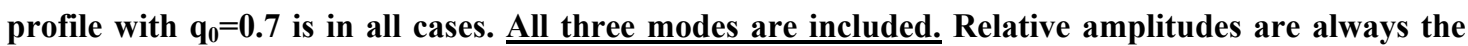
same as in figure 4. Absolute amplitudes are rescaled such that: (a) $(1,1)$ amplitude is $6 \mathrm{~cm}$; (b) $(1,1)$ amplitude is $4 \mathrm{~cm}$; (c) $(1,1)$ amplitude is $3 \mathrm{~cm}$; (d) $(1,1)$ amplitude is $2 \mathrm{~cm}$.

One can clearly see that the absence of $(2,2)$ and $(3,3)$ modes significantly increase the threshold of stochastization as shown in figure 11. To obtain the same degree of stochastization as shown in figure 10a for $6 \mathrm{~cm}$ displacement, in case of single $(1,1)$ mode, the amplitude of the $(1,1)$ mode has to be as large as $9-10 \mathrm{~cm}$. This clearly demonstrates the importance of inclusion of higher components. 

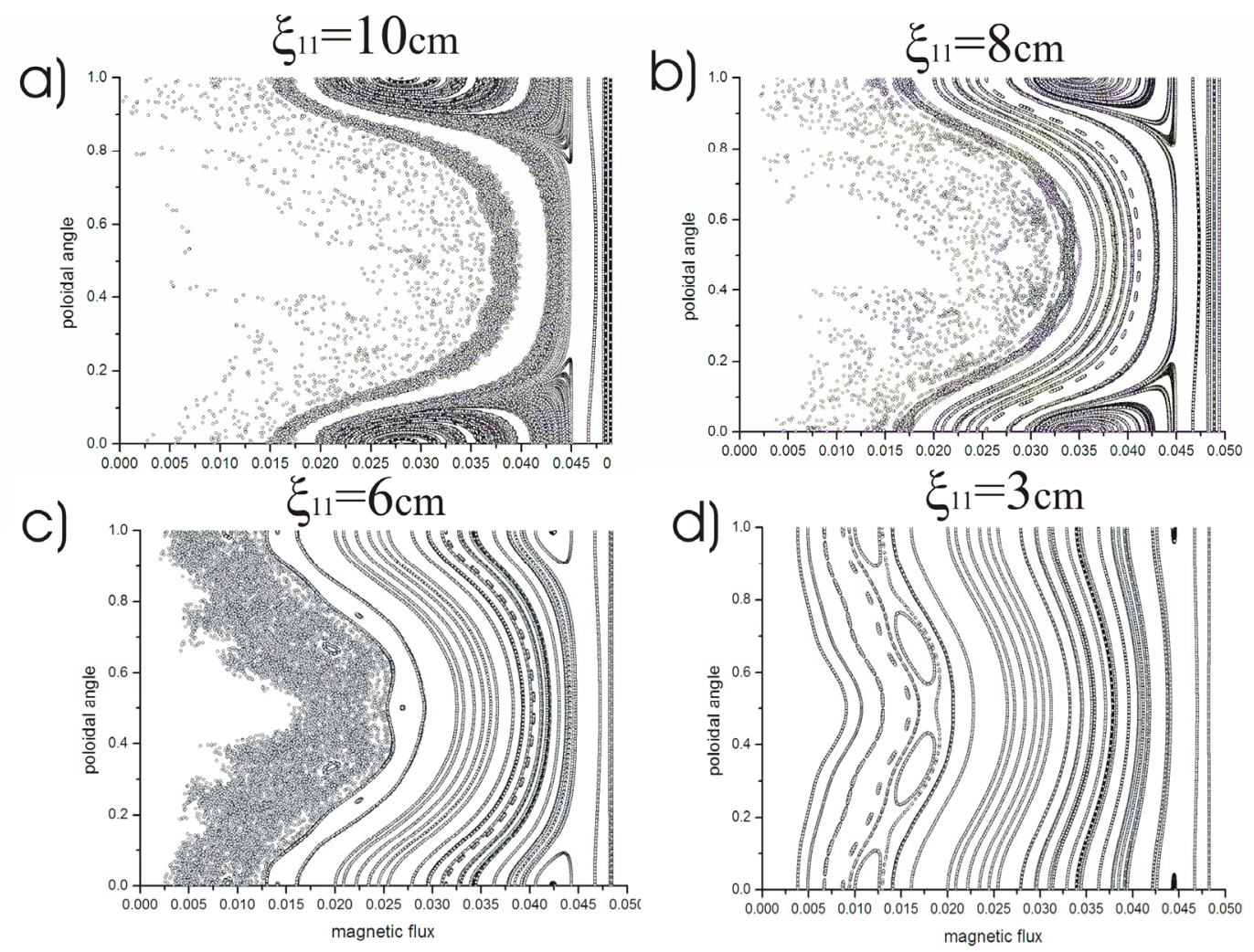

Figure 11. Influence of the absolute amplitude of the perturbations on stochasticity. Safety factor profile with $q_{0}=0.7$ is in all cases. Only the $(1,1)$ mode is included in the calculations. $(a)(1,1)$ amplitude is $10 \mathrm{~cm}$; (b) $(1,1)$ amplitude is $8 \mathrm{~cm}$; (c) $(1,1)$ amplitude is $6 \mathrm{~cm}$; (d) $(1,1)$ amplitude is 3 cm.

Dependence on the shear is also present. The shear determines a relative position of the low-order rational surfaces. However this dependence is not as important as the dependence on $q_{0}$ and on the perturbation amplitudes.

As was mentioned in the previous section, the absolute values of the $(1,1)$ mode displacement are about 5-6 cm. (The radius of the $\mathrm{q}=1$ rational surface is about 13-16 $\mathrm{cm}$.) Our calculations demonstrate that for these experimental perturbations the main parameter is $q_{0}$. In Table 1 we have summarized the excited resonant surfaces for different values of $q_{0}$. 
Table 1. Excited resonant surfaces for different values of the central safety factor $q_{0}$ are marked by stars. (*- resonance is excited. $\mathrm{n} / \mathrm{r}$ - not resolved due to high stochasticity. 0 - not present in the corresponding q-profile, blank places correspond to non excited resonances)

\begin{tabular}{|c|c|c|c|c|c|c|c|c|c|c|}
\hline $\mathrm{q}_{0} \backslash$ mode & $(3,4)$ & $(4,5)$ & $(5,6)$ & $(6,7)$ & $(7,8)$ & $(8,9)$ & $(9,10)$ & $(10,11)$ & $(11,12)$ & $(12,13)$ \\
\hline 0.70 & $*$ & $*$ & $*$ & $*$ & $*$ & $\mathrm{n} / \mathrm{r}$ & $\mathrm{n} / \mathrm{r}$ & $*$ & $\mathrm{n} / \mathrm{r}$ & $\mathrm{n} / \mathrm{r}$ \\
\hline 0.75 & 0 & $\mathrm{n} / \mathrm{r}$ & $*$ & $*$ & $*$ & $*$ & $*$ & $*$ & $*$ & $*$ \\
\hline 0.80 & 0 & 0 & $*$ & $*$ & $*$ & & & & $*$ & \\
\hline 0.85 & 0 & 0 & 0 & & & $*$ & & & & $*$ \\
\hline
\end{tabular}

It is evident that the resonant surfaces with the smallest possible poloidal mode numbers $(m=3,4,5,6)$ play the main role in creating stochastization. Excitation of such resonances requires significantly smaller perturbation amplitudes compared to the higher $m$ values. From mathematical point of view, equilibrium magnetic field in a tokamak is designed from close nested toroidal flux surfaces in real 3D vector space and this real space toroidal surface is the KAM surface in the 3D phase space of the Hamiltonian system for the magnetic field lines. In this formulation, the safety factor profile is the inversion of rotational transformation number in the chaos theory [20]. Thus, all formulations from chaos theory can be directly applied to the magnetic field structure. For our case this means excitation and destruction of the low order rational surfaces in safety factor profile, because in phase space all perturbations are coupled and low order rational surfaces are easily excited.

It is interesting to compare behaviour of the plasma core in our model with recently done 2D ECE measurements from TEXTOR [21,22] and results of nonlinear MHD simulation. The 2D ECE sawteeth measurements in TEXTOR show that the reconnection event has no preferential location along the poloidal magnetic surface and the heat flows like a fluid through the narrow gate in X-point. These observations match very well with our stochastic picture. According to our model, the sawtooth crash may occur at any poloidal position and the flow in the X-point is constrained by the confined island region so that the heat flows through the narrow channel (stochastic zone). This heat flow equalises temperature between the plasma core and region just outside $\mathrm{q}=1$ resonant surface. The mode amplitude decays during the crash phase which closes the 
heat channel in the X-point . The $(1,1)$ island structure is survived during the whole phase of the crash. This is different compared to nonlinear theory with secondary reconnection which requires: i) complete reconnection during which the $(1,1)$ mode is vanished completely; ii) secondary reconnection which creates the $(1,1)$ mode again $[23,24]$. The other, ballooning theory, suggests much broader region for the crash compared to the described models and preferable position at the low field side [25]. Both of these points are in contradictions with the 2D ECE measurements.

The ECE measurements also show that the temperature inside the $(1,1)$ island is smaller compared to the plasma core. This is also similar to the stochastic model which has hotter plasma in the stochastic region (plasma core) and opposite to the quasiinterchange model of the sawtooth [7].

\section{Conclusions}

In this paper we have investigated partial sawtooth reconnection in ASDEX Upgrade. The mapping technique was applied to trace the field lines of the toroidally confined plasma where perturbation parameters are expressed in terms of experimental perturbation amplitudes determined from the ASDEX Upgrade tokamak. The analysis shows that during a partial sawtooth reconnection the mode structure is complex and cannot be described by the $(1,1)$ component alone. The presence of a $(2,2)$ component in the analysis is very important. The third $(3,3)$ component is not so important as $(2,2)$, but it is necessary for correct representation of the experimental perturbations. It was demonstrated that an overlap of all three components is able to create a large stochastic region inside the $\mathrm{q}=1$ resonant surface leading to sawtooth crash. At the same time, the $(1,1)$ island structure itself is not stochastic which means that the mode survives the crash. (The island region becomes stochastic only for extremely large perturbations with displacement value higher than the Sawtooth inversion radius, which is impossible.) From mathematical point of view, this island structure survives as a result of the KAM theorem [26]. We have demonstrated that stochastization appears due to excitation of low- order resonances which are present in the corresponding q-profiles inside the $q=1$ surface which reflects the key role of the $q_{0}$ value. Depending on this value two completely different situations are possible for one and the same mode perturbations: (i) the resonant surfaces are present in q-profile leading to stochasticity and sawtooth crash 
( $q_{0} \approx 0.7 \pm 0.1$ ); (ii) the resonant surfaces are not present which means no stochasticity in the system and no crash event $\left(q_{0} \approx 0.9 \pm 0.05\right)$. It is remarkable that in both cases $q_{0}$ is lower than unity! Moreover, these values are in a good agreement with safety factor measurements performed on other tokamaks $(0.75-0.95)$. This result holds for variation of the perturbation amplitudes inside the error bars. Such variations only slightly change the critical values for $q_{0}$. Our model does not require the interaction of the fundamental $(1,1)$ mode with other periodicities, e.g., with $(1,0)$ external error field which is strongly screened by the plasma due to high plasma rotation. It was shown that the measured plasma perturbations are sufficient to explain the partial sawtooth reconnection in ASDEX Upgrade. Thus, the stochastic model agrees well with experimental observations and can be proposed as a promising candidate for explanation of the sawtooth reconnection. It is also clear that dynamic behaviour of the instability can not be described in the frame of the field line tracing approach and we plan further investigations of the Sawtooth crash with nonlinear MHD codes.

\section{Acknowledgments}

The authors are indebted to K. Itoh for helpful discussions about the Sawtooth phenomenon.

\section{References}

[1] S. Goeler, S. Stodiek, W. Sauthoff, Phys. Rev. Let. 33, 1201 (1974).

[2] A. Letsch, H. Zohm, F. Ryter, W. Suttrop, A. Gude, F. Porcelli, C. Angioni and I. Furno, Nucl. Fusion 42, 1055 (2002).

[3] V. S. Udintsev et.al., Plasma Physics and Control Fusion 47, 1111 (2005).

[4] S. Annibaldi, P. Buratti, E. Giovannozzi and F. Zonca, Plasma Physics and Control Fusion 47, 1367 (2005).

[5] V. Igochine, S. Guenter, M. Maraschek and the ASDEX Upgrade Team., Nucl. Fusion 43, 1801 (2003).

[6] B. B. Kadomtsev, Soviet Journal of Plasma Physics, 1, 710 (1975).

[7] J. A. Wesson, Plasma Physics and Control Fusion 28(1A), 243 (1986).

[8] K. Itoh, S-I. Itoh, and A. Fukuyama, Plasma Phys. Control. Fusion 37, 1287 (1995).

[9] A. J. Lichtenberg, Nucl. Fusion 24, 1277 (1984). 
[10] A. J. Lichtenberg, K. Itoh, S.-I. Itoh, A. Fukuyama , Nucl. Fusion 32, 495 (1992).

[11] J. S. Kim, M. S. Chance, D.H. Edgell, J.M. Greene, E.J. Strait and A.D. Turnbull, Plasma Physics and Control Fusion 43, 1593 (2001).

[12] K. Ertl, W. von der Linden, V. Dose, A. Weller, Nucl. Fusion 36, 1477 (1996).

[13] M. Socoll, IPP report (IPP 1/309) April 1997.

[14] S.S. Abdullaev, „Construction of Mappings for Hamiltonian Systems and Their Applications”, Lect. Notes Phys. 691 (Springer, Berlin, Heidelberg, 2006).

[15] I. Pavlenko, B. Rapoport, B. Weyssow and D.Carati, Physics of Plasmas, 10, 1083 (2003).

[16] S.S. Abdullaev, Nucl. Fusion 44, S12 (2004).

[17] F.M. Levinton, L. Zakharov, S.H. Batha, J. Manickam, M.C. Zarnstorff, Phys.Rev.Lett.,72,2895 (1994).

[18] M.Yamada, F.M. Levinton, N. Pomphrey, R.Budny, J.Manickam, Y. Nagayama, Physics of Plasmas, 1, 3269 (1994).

[19] H.Soltwisch, Rev. Sci. Inst., 59, 1599 (1988).

[20] W. Horton and Y.-H. Ichikawa, „Chaos and structures in nonlinear plasmas”, World Scientific Publishing, Singapore, reprinted in 2000 (ISBN 9810226365).

[21] H.K. Park, E. Mazzucato, N.C. Luhmann, Jr., C.W. Domier, Z. Xia, T. Munsat, A.J.H. Donne, I.G.J. Classen, M.J. van de Pol and TEXTOR team, Physics of Plasmas, 13, 55907 (2006).

[22] H.K. Park, N.C. Luhmann, Jr., A.J.H. Donne, I.G.J. Classen, C.W. Domier, E. Mazzucato, T. Munsat, M.J. van de Pol, Z. Xia, and TEXTOR team, Phys. Rev. Let. 96, 195003 (2006).

[23] D. Biskamp, J. F. Drake,Phys. Rev. Let. 73, 971 (1994).

[24] A. Ishizawa, M. Sato, M. Wakatani, Physics of Plasmas, 10, 3017 (2003).

[25] Y. Nishimura, J. D. Callen, and C. C. Hegna, Physics of Plasmas, 6, 4685 (1999).

[26] V. I. Arnold, Mathematical Methods of Classical Mechanics (Springer-Verlag, New York, 1978). 